\title{
Analysis of Cotton Chromosome 11 and 14 Root-Knot Nematode Resistance Quantitative Trait Loci Effects on Root-Knot Nematode Postinfection Development, Egg Mass Formation, and Fecundity
}

\author{
Martin J. Wubben, ${ }^{\dagger}$ Amanda G. Gaudin, Jack C. McCarty, Jr., and Johnie N. Jenkins \\ Genetics and Sustainable Agriculture Research Unit, U.S. Department of Agriculture Agricultural Research Service, Mississippi State, \\ MS 39762 \\ Accepted for publication 27 December 2019.
}

\begin{abstract}
Cotton (Gossypium hirsutum) resistance to root-knot nematode (RKN) (Meloidogyne incognita) is controlled by quantitative trait loci (QTLs) on chromosomes 11 (CHR11) and 14 (CHR14). The individual contributions of these QTLs to resistance are not completely understood. We developed near isogenic lines susceptible at both loci (null), having CHR11 or CHR14 alone, and having both QTLs (CHR11/CHR14). RKN reproduction, postinfection development, egg mass formation, and adult female fecundity were evaluated. Total RKN reproduction was reduced more in CHR14 versus CHR11 but not as greatly as in CHR11/CHR14. Secondstage juvenile (J2) development to the $\mathrm{J} 3$ and $\mathrm{J} 4(\mathrm{~J} 3+\mathrm{J} 4)$ life stages was delayed in CHR11, whereas the $\mathrm{J} 2$ transition to J3+J4 in CHR14 followed a similar track as in null plants. Development of J3+J4
\end{abstract}

ABSTRACT nematodes to adult females was inhibited in CHR14 at 21 days after inoculation (DAI). Adult female numbers were decreased in CHR11 and CHR14 at 21 and 28 DAI, with CHR11/CHR14 showing an even greater reduction by 28 DAI. The number of egg masses per gram of root at 21 , 28, and 35 DAI formed on CHR11 and CHR14 followed a similar track as numbers of adult females. RKN adult female fecundity (eggs/egg mass) was reduced for CHR11 and CHR14 compared with the null at 21 DAI; however, CHR11 eggs/egg mass was only slightly reduced versus the null by 28 DAI. In contrast, CHR14 eggs/egg mass was like CHR11/ CHR14, showing a 4-fold decrease compared with CHR11 and the null.

Keywords: genetics, resistance
The southern root-knot nematode (RKN) (Meloidogyne incognita Kofoid \& White) is a sedentary endoparasite that has long been a major pest of Upland cotton (Gossypium hirsutum L.) throughout the United States. For example, in 2016, cotton losses attributable to RKN infection were valued at more than $\$ 130$ million (Lawrence et al. 2017). The severe root galling that occurs because of RKN infection disrupts water and nutrient transport in the root system, resulting in reduced plant growth, reduced leaf area, and fewer bolls (Kirkpatrick et al. 1995; Lu et al. 2014). In the absence of effective host resistance, management of RKN in cotton production depends largely on the application of nematicides that are relatively expensive and do not provide protection throughout the growing season. The planting of cotton cultivars with a high level of natural host resistance to RKN would be the most costeffective and environmentally sustainable solution for producers.

Cotton infection by RKN is initiated when eggs in the soil or associated with infected root systems hatch and release infective second-stage juveniles (J2). Infection occurs primarily at the root tips and zone of elongation and is facilitated by the secretion of a wide assortment of plant cell wall-degrading enzymes that allow the $\mathrm{J} 2$ to penetrate the root epidermis and migrate intercellularly through the root (Mitchum et al. 2013; Williamson and Hussey 1996). The J2 become sedentary and establish a feeding site near

†Corresponding author: M. J. Wubben; martin.wubben@usda.gov

Mention of trade names or commercial products in this publication is solely for the purpose of providing specific information and does not imply recommendation or endorsement by the U.S. Department of Agriculture (USDA). USDA is an equal opportunity provider and employer.

The author(s) declare no conflict of interest.

This article is in the public domain and not copyrightable. It may be freely reprinted with customary crediting of the source. The American Phytopathological Society, 2020. the root vascular cylinder. The feeding site consists of five to eight "giant cells" that function as a sugar sink for the plant and nutrient source for the nematode (Williamson and Hussey 1996). Giant cell initiation, development, and maintenance is controlled by a myriad of effector proteins that are secreted by the developing nematode (Mitchum et al. 2013). The characteristic RKN-induced root galls form in concert with feeding site formation and are attributable to cortical cell hyperplasia (Williamson and Hussey 1996). The establishment and maintenance of the feeding site is critical to the RKN juvenile in order to successfully molt from the $\mathrm{J} 2$ through the $\mathrm{J} 3$ and $\mathrm{J} 4$ life stages and end as a mature female. M. incognita reproduces via mitotic parthenogenesis with the female depositing eggs within a gelatinous matrix on the surface of the root (Williamson and Hussey 1996).

A high level of Upland cotton resistance to RKN was first identified in a transgressive segregant from a cross between the moderately resistant accessions Clevewilt-6 and Mexico Wild Jack Jones and later released as the Auburn 623 RNR germplasm line (Shepherd 1974). A study of RKN infection and symptom progression in Auburn 623, compared with the susceptible line M-8, showed that egg mass development on Auburn 623 was reduced by $92 \%$ and the number of eggs contained within the egg masses was reduced by $75 \%$ compared with M-8 (Shepherd and Huck 1989). The Auburn 623 resistance was successfully transferred into several germplasm lines with better agronomic traits, including the "M-series" of lines such as M-240 RNR and others (Shepherd 1982; Shepherd et al. 1996). Genetic studies suggested that Auburn 623-derived resistance was controlled by at least two genes that behaved in a dominant and additive fashion (McPherson et al. 1995, 2004). In a series of mapping studies using $\mathrm{F}_{2}$ and recombinant inbred line (RIL) populations, it was determined that Auburn 623-derived resistance was mediated by two major quantitative trait loci (QTLs) that resided on cotton chromosomes 11 (CHR11) and 14 (CHR14) (Gutiérrez et al. 2010; Shen et al. 2006; Ynturi et al. 2006). These QTLs, referred to as $q M i$ $\mathrm{Cl1}$ and $q \mathrm{Mi}-\mathrm{Cl}$, differed in how they contributed to the RKN 
resistance phenotype. Although both QTLs reduced overall RKN egg production, $q M i-C 11$ also significantly reduced gall development (Gutiérrez et al. 2010; He et al. 2014; Shen et al. 2006). In contrast, qMi-C14 did not impact gall development but showed a greater negative effect on egg production compared with $q M i-C 11$ (Gutiérrez et al. 2010; He et al. 2014). Although the causal gene underlying $q M i$ C11 remains unknown, a 2019 study used a combination of RIL population genome sequencing and virus-induced gene silencing and identified Gh_D02G0276 as the causal gene mediating resistance conferred by $q M i-C 14$ (Wubben et al. 2019).

The first investigations into the mechanistic aspects of Auburn 623-derived resistance were conducted prior to the identification of $q \mathrm{Mi}-\mathrm{Cl1}$ and $q \mathrm{Mi}-\mathrm{Cl}$; nevertheless, these studies revealed critical insights into how resistance was manifested. Using the Auburn 623derived germplasm line M-315 RNR, it was demonstrated that resistance did not affect the ability of the RKN J2 to penetrate the root but greatly affected egg mass formation and eggs per egg mass (Creech et al. 1995). In a parallel study, RKN development was monitored over time in M-315 RNR plants compared with the susceptible control M-8. From this study, the authors concluded that an "early" and "late" stage of resistance was evident that inhibited both feeding site formation by the $\mathrm{J} 2$ and maturation of those nematodes that were able to establish feeding sites (Jenkins et al. 1995). Using the Auburn 623-derived germplasm line M-120 RNR, the effects of $q \mathrm{Mi}-\mathrm{Cl1}$ and $q \mathrm{Mi}-\mathrm{Cl} 4$ on RKN development were reported (Da Silva et al. 2019). From this study, Da Silva et al. (2019) concluded that $q M i-C 11$ significantly reduced nematode development beyond the $\mathrm{J} 2$ stage, whereas development beyond the $\mathrm{J} 3 / \mathrm{J} 4$ life stage was inhibited by $q \mathrm{Mi}-\mathrm{C} 14$. Although the study by $\mathrm{Da}$ Silva et al. (2019) was thorough in its assessment of postinfection RKN development, the individual effects of $q \mathrm{Mi}-\mathrm{Cl} 1 \mathrm{l}$ and $q \mathrm{Mi}-\mathrm{Cl} 4$ on egg mass formation and fecundity were not reported.

In this report, we provide a comprehensive assessment of the

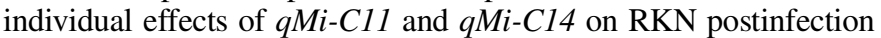
development, egg mass formation, and adult female fecundity. This work was accomplished using near isogenic lines (NILs) developed from the Auburn 623-derived germplasm line M-240 RNR that carried $q \mathrm{Mi}-\mathrm{Cl1}$ and $q \mathrm{Mi}-\mathrm{Cl} 4$ in isolation, carried both QTLs together, or were null for both loci. Our findings complete the overall picture of how $q M i-C 11$ and $q M i-C 14$ manifest RKN resistance and demonstrate how these QTLs differ in their timing of effect on RKN development and in their impact on female fecundity.

\section{MATERIALS AND METHODS}

Development of RKN resistance QTL NILs. The RKNresistant germplasm line M-240 RNR (Shepherd et al. 1996) was crossed to RKN-susceptible FiberMax-966 (FM966). Four backcrosses were made to FM966 to create the $\mathrm{BC}_{4} \mathrm{~F}_{1}$. The $\mathrm{BC}_{4} \mathrm{~F}_{1}$ was selfed to create a $\mathrm{F}_{2}$ population segregating for $q \mathrm{Mi}-\mathrm{Cl} 1 \mathrm{and} q \mathrm{Mi}$ $C 14 . \mathrm{F}_{2}$ plants were identified that were susceptible for both QTLs (null), homozygous for $q M i-C 11$ (CHR11), homozygous for $q M i$ C14 (CHR14), and homozygous for both QTLs (CHR11/CHR14). $\mathrm{F}_{2}$ genotyping was performed on genomic DNA isolated from leaf tissue samples using the molecular markers CIR316 for $q M i-C 11$ and BNL3661 for $q M i-C 14$ as previously described (Gutiérrez et al. 2010; Jenkins et al. 2012). Selfed seed from selected $F_{2}$ plants was planted and the resulting $\mathrm{F}_{2: 3}$ plants were genotyped using the same molecular markers to confirm the original $\mathrm{F}_{2}$ designation. Genotype-confirmed $\mathrm{F}_{2: 3}$ plants were selfed and the resulting seed was used for subsequent experiments.

Nematode inoculum. A southern RKN (M. incognita) population was propagated on susceptible cotton in growth chambers. Eggs used for inoculum in experiments were collected from roots of infected plants by agitation in $1 \%$ sodium hypochlorite for 3 min and collected on a $25-\mu \mathrm{M}$ sieve as described by Hussey and Barker (1973). J2 used for inoculum in experiments were hatched from eggs collected as described above. Briefly, RKN eggs were layered over a $25-\mu \mathrm{M}$ nylon mesh and incubated in water at $30^{\circ} \mathrm{C}$. Hatched $\mathrm{J} 2$ were collected at daily intervals for 7 to 10 days.

Evaluation of NIL susceptibility to RKN. Four NILs of the null, CHR11, CHR14, and CHR11/CHR14 genotypes were evaluated for RKN susceptibility. Seeds were scarified and imbibed at $30^{\circ} \mathrm{C}$ for $4 \mathrm{~h}$ before being wrapped in moist paper towels and incubated overnight at $30^{\circ} \mathrm{C}$ to promote germination. Germinated seeds having radicles of similar length were planted into an autoclaved soil mixture of one part Wickham sandy loam to two parts sand, supplemented with five to eight Osmocote beads (ScottsSierra Horticultural Products Co., Marysville, OH), contained within 20.32-cm Cone-tainers (Stuewe \& Sons, Tangent, OR). Upon seedling emergence ( 1 to 2 days after planting), each Conetainer was inoculated with approximately 5,000 RKN eggs administered as $2 \times 1-\mathrm{ml}$ aliquots injected into 5-cm-deep holes made on opposite sides of the seedling. Plants were grown at $30^{\circ} \mathrm{C}$ (16 h of light/8 h of dark) in a Percival PGC-9/2 growth chamber (Percival Scientific, Perry, IA). Six weeks after inoculation, soil was gently washed from the roots of each plant. The roots of three plants were pooled to form one experimental replication (five replications per isoline per experiment), blotted dry, and weighed to determine the fresh weight (in grams). Eggs were extracted from each pooled sample of three root systems as described above and collected into 140-ml specimen cups. Eggs were stained by adding $1 \mathrm{ml}$ of acid fuchsin to each sample and were then heated to boiling in a microwave. The total volume of the egg sample was made $100 \mathrm{ml}$, from which $1 \mathrm{ml}$ was taken and counted using a stereomicroscope.

Determination of RKN development, egg mass formation, and fecundity. To track RKN development over time, seedlings ( 2 to 3 days after planting) were inoculated with approximately 1,600 J2 administered as $4 \times 1-\mathrm{ml}$ aliquots injected into holes spaced evenly around the perimeter of the Cone-tainer. Ten plants of each NIL were collected at 7, 14, 21, and 28 days after inoculation (DAI). After being washed free of soil, the root system of each plant was cleared by soaking in $1 \%$ sodium hypochlorite for 3 to $4 \mathrm{~min}$. Roots were rinsed with tap water for approximately $1 \mathrm{~min}$ on a $25-\mu \mathrm{M}$ sieve and then soaked in tap water for at least $15 \mathrm{~min}$ to remove any residual sodium hypochlorite. Roots were transferred to specimen cups to which $60 \mathrm{ml}$ of tap water plus $2 \mathrm{ml}$ of acid fuchsin was added. The root/staining solution was boiled for 1 min using a microwave. After cooling to room temperature, roots were rinsed for 1 min with tap water, blotted dry, and transferred to $140-\mathrm{ml}$ beakers. Roots were suspended in approximately $25 \mathrm{ml}$ of acidified glycerin for storage. Stained roots were placed in-between glass plates and RKNs were visualized and counted with a stereomicroscope.

For egg mass counting, J2-inoculated seedlings were collected at 21, 28, and 35 DAI. Roots were gently washed free of soil and egg masses were stained by incubating the roots in a $0.25 \%$ trypan blue (wt/vol) solution for $20 \mathrm{~min}$ at room temperature. Roots were destained by soaking in tap water. Egg masses were then counted using a stereomicroscope. After counting, roots were blotted dry and the fresh weight (in grams) was determined. Fresh weight was determined after counting to prevent dislodgement of egg masses during blotting with paper towels.

To determine RKN fecundity, seedlings were inoculated with approximately 1,500 J2s as described above. At 21 and $28 \mathrm{DAI}$, five plants each of null, CHR11, and CHR14 and 10 plants of CHR11/ CHR14 were collected and the roots were gently washed free of soil. Root systems of all plants for each NIL were cut into 1- to 2-cm pieces and combined in a 250-ml beaker. Egg masses were stained with $0.25 \%$ trypan blue and root pieces were destained as described above. Fifteen egg masses were dissected from randomly selected root pieces for each NIL. Egg masses that clearly originated from a single female were selected. To determine the egg number, an egg mass was transferred to a small Petri dish containing 1 to $2 \%$ sodium hypochlorite. Dissolution of the gelatinous matrix was observed using a stereomicroscope and facilitated by teasing the egg mass apart using a forceps and scalpel. All eggs were counted once freed from the egg mass. 
Statistical analyses. All data were subjected to an analysis of variance using PROC GLM in SAS software (version 9.3; SAS Institute). Means were separated using Fisher's protected least significant difference at $P \leq 0.05$.

\section{RESULTS}

Effects of $q M i-C 11$ and $q M i-C 14$ on RKN reproduction. Upland cotton NILs were developed that expressed $q M i-C 11$ alone (CHR11), qMi-C14 alone (CHR14), both QTLs (CHR11/CHR14), or neither QTL (null). Four NILs of each genotype were evaluated for total RKN reproduction in growth chamber experiments (Table 1). Null genotypes showed a range of susceptibilities with average values of 31,061 to 81,314 eggs per gram of root. At the other end of the spectrum, the four CHR11/CHR14 NILs grouped closely together with mean values of 388 to 783 eggs per gram of root. CHR11 NILs showed averages of 17,525 to 43,511 eggs per gram of root, whereas CHR14 NILs showed averages less than 10,000 eggs per gram of root except for line 13-63-45-GH-139 (mean = 23,691). NILs GH-178 (null), GH-80 (CHR11), GH-152 (CHR14), and GH-44 (CHR11/CHR14) showed relative susceptibilities consistent with previous experiments (Gutiérrez et al. 2010; Jenkins et al. 2012) and exhibited the lowest standard error values (Table 1) within their genotype groups; therefore, these NILs were used for future experiments.

Temporal effects of $q M i-C 11$ and $q M i-C 14$ on RKN development. The effects of $q M i-C 11$ and $q M i-C 14$ on RKN penetration and postinfection development were determined at 7 , 14, 21, and 28 DAI. Migratory J2, sedentary J2, J3, and J4, and adult females were counted in five to eight plants each of acid fuchsin-stained roots of null, CHR11, CHR14, and CHR11/CHR14 plants.

$R K N$ migratory and sedentary $J 2$. For ease of analysis and interpretation, migratory and sedentary $\mathrm{J} 2$ numbers were combined. At 7 DAI, roots of null and CHR14 plants showed slightly fewer numbers of J2s than CHR11 and CHR11/CHR14 plants (Fig. 1A); however, when converted to a percentage of total nematodes counted, there was no difference in $\mathrm{J} 2$ infection between genotypes at 7 DAI (Fig. 1B). At 14 DAI, the total number of $\mathrm{J} 2$ decreased significantly for each genotype; however, this decrease was more pronounced for null and CHR14 versus CHR11 and CHR11/ CHR14 as shown by raw count and percentage data (Fig. 1A and B). For null and CHR14 plants, the total number of J2 showed no further decrease between 14, 21, and 28 DAI (Fig. 1A). In contrast, CHR11 and CHR11/CHR14 plants showed another significant decrease in $\mathrm{J} 2$ numbers between 14 and 21 DAI that was also reflected in the percentage data (Fig. 1A and B).

$R K N \mathrm{~J} 3+\mathrm{J} 4$ juvenile stages. Numbers of $\mathrm{J} 3$ and $\mathrm{J} 4$ nematodes were combined for ease of analysis and interpretation. As expected, at $7 \mathrm{DAI}$, few nematodes were observed at these more advanced life stages in each of the genotypes studied (Fig. 1C). However, J3+J4 values increased tremendously by $14 \mathrm{DAI}$ for each genotype. At 14 DAI, null and CHR14 plants showed J3+J4 levels that were significantly greater than those found in roots of CHR11 and CHR11/CHR14 plants (Fig. 1C). The 7 and 14 DAI count data trends were mirrored almost exactly when converted to a percentage of total nematodes (Fig. 1D). At 21 DAI, raw counts of $\mathrm{J} 3+\mathrm{J} 4$ nematodes were equivalent for all genotypes with values decreasing from 14 DAI for null, CHR11, and CHR14 (Fig. 1C). However, $\mathrm{J} 3+\mathrm{J} 4$ values as a percentage of total numbers showed clear differences between genotypes at 21 DAI (Fig. 1D). J3+J4 nematodes comprised significantly less of the total nematode population in roots of null plants versus CHR11, CHR14, and CHR11/CHR14 (Fig. 1D). In addition, CHR14 and CHR11/CHR14 plants showed similar J3+J4 percentage values at 21 DAI (Fig. 1D). By 28 DAI, J3+J4 counts had decreased significantly for all genotypes and were not significantly different from each other (Fig. 1C). This observation largely held true with the percentage data, with only CHR11/CHR14 plants showing an elevated proportion of $\mathrm{J} 3+\mathrm{J} 4$ in roots versus the other genotypes (Fig. 1D).

RKN females. Mature RKN females were first observed at 14 DAI with no significant differences in count data being detected between genotypes (Fig. 1E); however, mature females comprised a greater percentage of the total nematode population in null plant roots versus resistant genotypes at this timepoint (Fig. 1F). Null plants showed a substantial increase in female numbers by 21 DAI (Fig. 1E). In contrast, female numbers did not change significantly between 14 and 21 DAI for CHR11 and CHR11/CHR14 plants (Fig. $1 \mathrm{E})$. At $21 \mathrm{DAI}$, mature females comprised nearly $73 \%$ of all nematodes in null plants compared with CHR11 (36\%), CHR14 (59\%), and CHR11/CHR14 plants (26\%) (Fig. 1F). Female numbers leveled off between 21 and 28 DAI for all genotypes (Fig. 1E); however, female percentages were $>83 \%$ for null, CHR11, and CHR14 plants, whereas CHR11/CHR14 showed a similar percentage value to $21 \mathrm{DAI}$ (Fig. 1F).

Effects of $q M i-C 11$ and $q M i-C 14$ on RKN egg mass formation. To gain additional insight into the effects of $q M i-C 11$ and $q M i-C 14$ on $M$. incognita development and reproduction, egg mass formation was evaluated on plants at 21, 28, and 35 DAI. At 21 DAI, the mean egg mass per gram of root was similar between null, CHR11, and CHR14 plants; however, significantly fewer egg masses were observed on CHR11/CHR14 plants versus the null (Fig. 2). By 28 DAI, a clear distinction between resistant and null plants was observed, with CHR11, CHR14, and CHR11/CHR14 plants showing means significantly less than the null isoline (Fig. 2). In addition, although the CHR14 and CHR11/CHR14 means were similar, the 28 DAI mean for CHR11 was significantly greater than that for CHR11/CHR14 (Fig. 2). Finally, at 35 DAI, a clear partitioning between all groups was observed, with CHR11 and CHR14 being similar to each other yet significantly less than the null but greater than when combined in CHR11/CHR14 (Fig. 2).

Across timepoints, null plants behaved differently from all resistant genotypes in that the mean egg mass per gram of root increased at each successive timepoint (Fig. 2). In contrast to the null, both CHR11 and CHR14 plants only showed a significant increase in egg mass number between 21 and 35 DAI (Fig. 2). When combined in CHR11/CHR14, means remained static across all timepoints with no significant gain in egg mass numbers after 21 DAI (Fig. 2).

Effects of $q M i-C 11$ and $q M i-C 14$ on $\mathrm{RKN}$ adult female fecundity. RKN fecundity, as measured by eggs/egg mass, was determined at 21 and 28 DAI for null, CHR11, CHR14, and CHR11/ CHR14 plants. Eggs/egg mass counts were attempted at 35 DAI; however, significant $\mathrm{J} 2$ hatch had occurred by this timepoint,

TABLE 1. Susceptibility of cotton resistance quantitative trait loci (QTLs) near isogenic lines (FiberMax-966 background) to root-knot nematode (RKN)

\begin{tabular}{llc}
\hline Line & RKN QTL & Eggs per gram of root $^{\mathrm{b}}$ \\
\hline 13-63-45-GH-131 & Null & $31,061 \pm 10,165$ \\
13-63-45-GH-174 & Null & $57,092 \pm 3,161$ \\
13-63-45-GH-177 & Null & $64,332 \pm 10,732$ \\
13-63-45-GH-178 & Null & $81,314 \pm 2,688$ \\
13-63-45-GH-35 & CHR11 & $43,511 \pm 7,372$ \\
13-63-45-GH-80 & CHR11 & $17,525 \pm 2,452$ \\
13-63-45-GH-145 & CHR11 & $29,313 \pm 5,748$ \\
13-63-45-GH-160 & CHR11 & $34,314 \pm 6,384$ \\
13-63-45-GH-127 & CHR14 & $3,448 \pm 2,319$ \\
13-63-45-GH-139 & CHR14 & $23,691 \pm 4,234$ \\
13-63-45-GH-152 & CHR14 & $9,306 \pm 1,935$ \\
13-63-45-GH-170 & CHR14 & $3,946 \pm 2,937$ \\
13-63-45-GH-1 & CHR11/CHR14 & $388 \pm 245$ \\
13-63-45-GH-44 & CHR11/CHR14 & $488 \pm 188$ \\
$13-63-45-G H-130$ & CHR11/CHR14 & $783 \pm 236$ \\
13-63-45-GH-182 & CHR11/CHR14 & $504 \pm 350$ \\
FiberMax-966 & Null & $95,321 \pm 18,768$ \\
M-240 RNR & CHR11/CHR14 & $1,277 \pm 1,169$ \\
\hline
\end{tabular}

a $\mathrm{CHR} 11=$ chromosome 11 and CHR14 = chromosome 14.

b Values are presented as the mean \pm SE. 


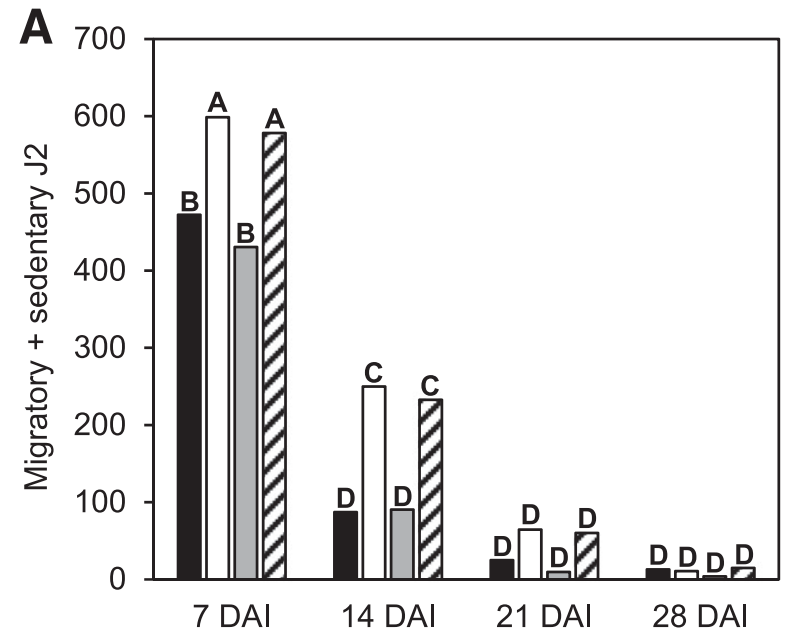

-Null aCHR11 घCHR14 घCHR11/CHR14

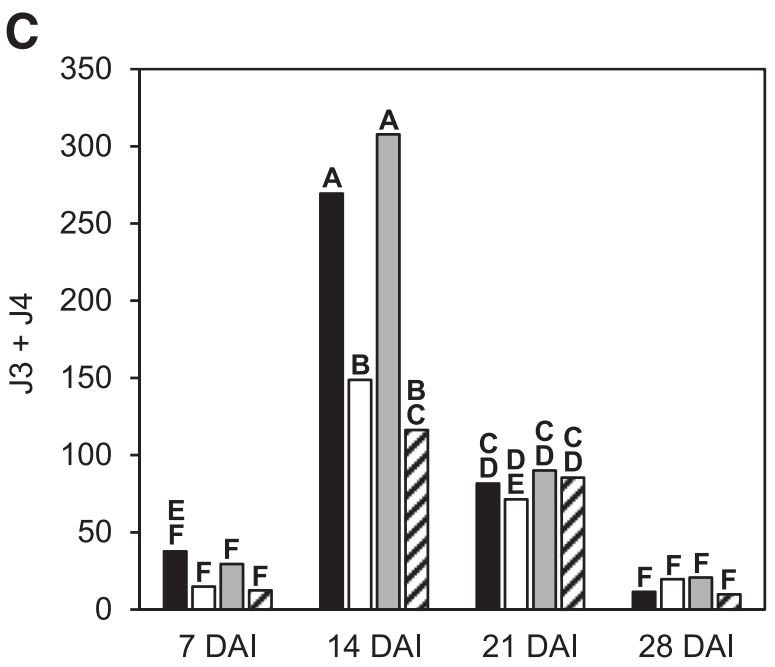

- Null aCHR11 aCHR14 चCHR11/CHR14

E

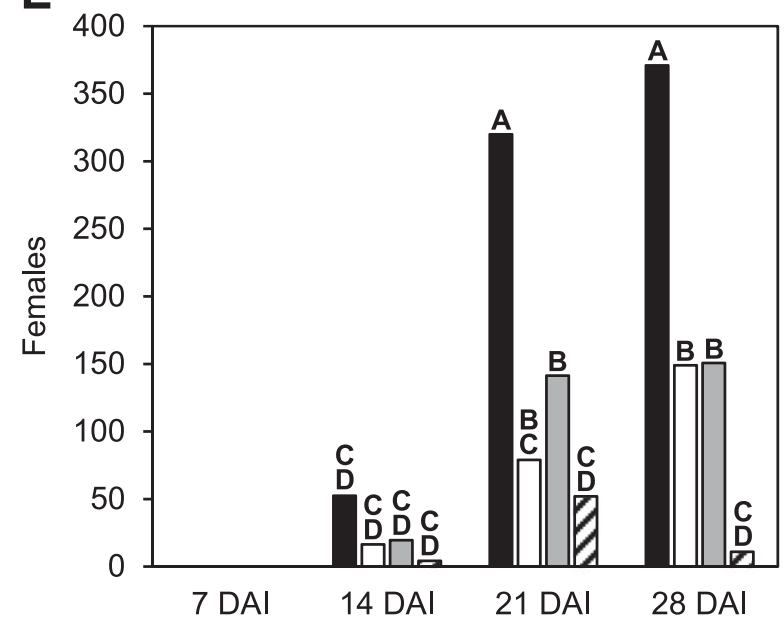

a Null aCHR11 aCHR14 aCHR11/CHR14

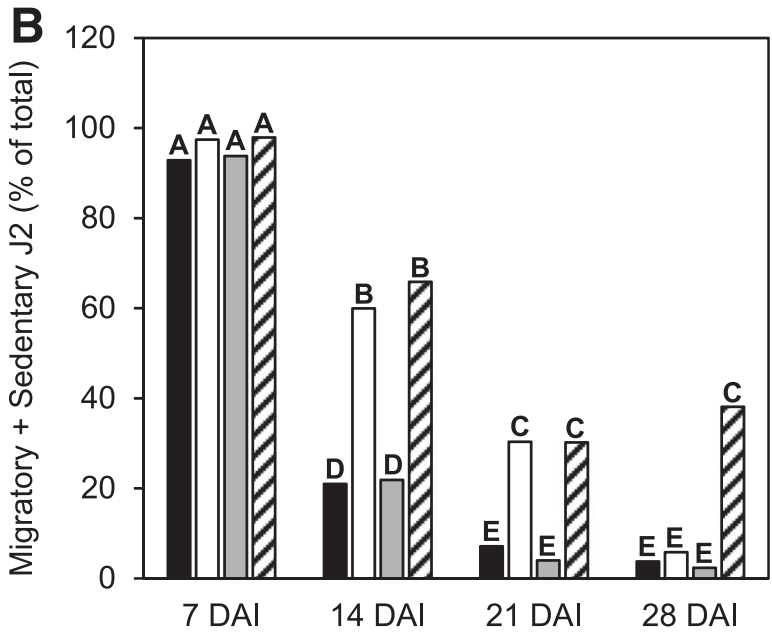

anull $\square$ CHR11 $\square \mathrm{CHR} 14 \quad \square \mathrm{CHR} 11 / \mathrm{CHR} 14$

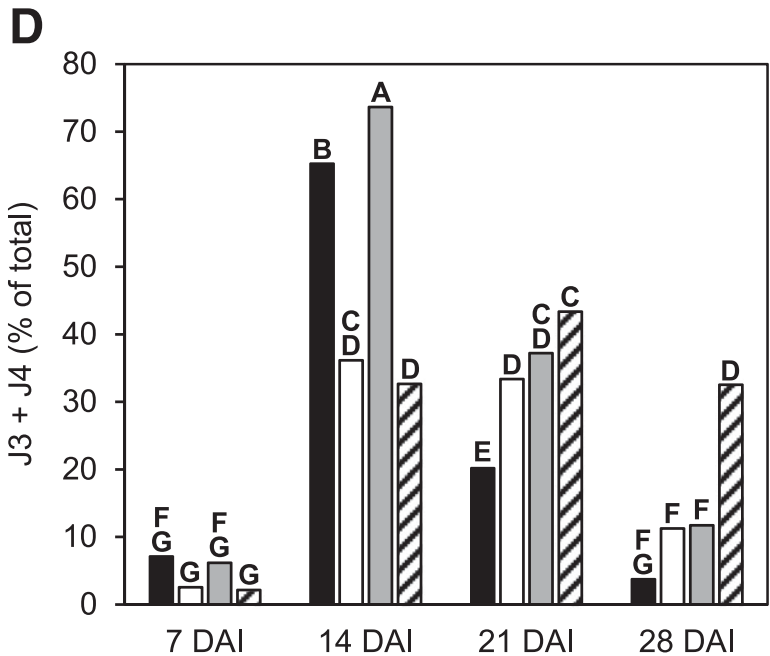

a Null aCHR11 aCHR14 aCHR11/CHR14

$\mathbf{F}$

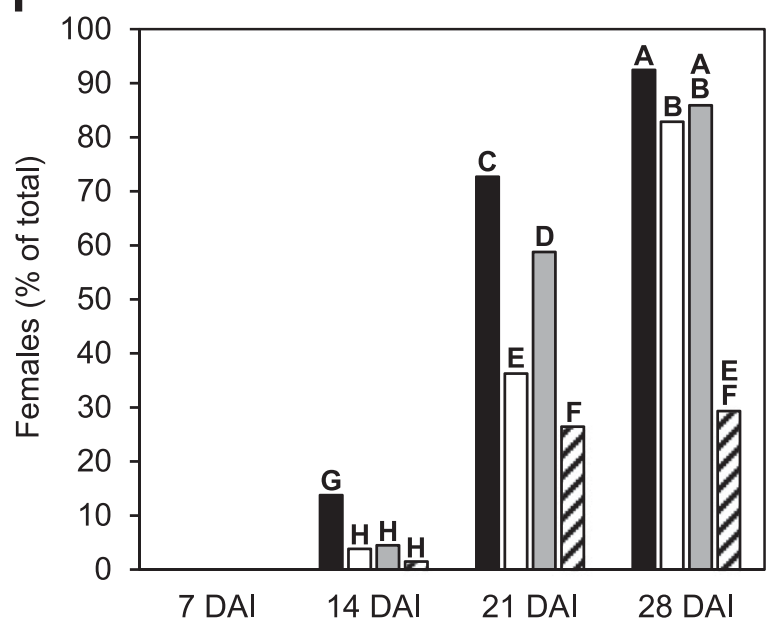

- Null aCHR11 aCHR14 aCHR11/CHR14

Fig. 1. Tracking of root-knot nematode (RKN) development in near isogenic lines (FiberMax-966 background) that are susceptible for $q M i-C 11$ and $q M i-C 14$ (null), carry qMi-C11 alone (chromosome 11 [CHR11]), carry qMi-C14 alone (chromosome 14 [CHR14]), and carry both quantitative trait loci (CHR11/CHR14). Presented are the mean ( $n=8$ plants) number of RKN juveniles in the A, second life stage (J2) (migratory + sedentary J2), C, third and fourth life stages (J3+J4), and $\mathbf{E}$, adult female life stage as determined at 7, 14, 21, and 28 days after inoculation (DAI). B, D, and F, Also presented are the same life stage categories expressed as a percentage of the total nematodes counted. Means having the same letter designation are not significantly different as determined using Fisher's protected least significant difference at $P \leq 0.05$. 
rendering accurate egg counts impossible (data not shown). At 21 DAI, egg masses formed on null plants contained more eggs than any of the resistant genotypes (Fig. 3). Egg masses formed on CHR11/CHR14 plants contained the fewest number of eggs but this mean was only significantly different from null and CHR11 plants (Fig. 3). By 28 DAI, eggs/egg mass means increased significantly for each genotype compared with 21 DAI, with the greatest increases having occurred on null and CHR11 plants (Fig. 3). At 28 DAI egg masses collected from null and CHR11 plants contained nearly two to four times the number of eggs found in egg masses formed on CHR14 and CHR11/CHR14 plants (Fig. 3).

\section{DISCUSSION}

The high level of RKN resistance derived from Auburn 623 RNR located on cotton CHR11 and CHR14 is a critical resource for U.S. cotton producers. In 2019, an estimated $6.7 \%$ of total cotton acreage was planted with varieties carrying $q M i-C 11$ and $q M i-C 14$, equivalent to 924,000 acres (U.S. Department of Agriculture 2019). The durability and longevity of these resistance QTLs depends on a thorough understanding of how they work together to bring about a high level of resistance to RKN. Using marker-assisted selection, we developed NILs that carried $q M i-C 11$ and $q M i-C 14$ in isolation such that their specific effects on RKN reproduction, postinfection development, egg mass formation, and adult female fecundity could be studied over time.

The absence of CHR11 and CHR14 gene-specific markers at the time of NIL development and reliance on the closely linked markers CIR316 and BNL3279, respectively, for genotype selection raised the possibility of selecting plants having recombinations between the marker and the resistance locus that would lead to false-positive and false-negative selections. Furthermore, the presence or absence of loci with minor effects coming from the M-240 RNR parent in the null, CHR11, and CHR14 genotypes would be another potential source of phenotypic variation. For these reasons, we evaluated multiple NILs of each genotype for total RKN reproduction to ensure that lines used in subsequent experiments were as close to being true null, CHR11, CHR14, and CHR11/CHR14 genotypic representatives as possible. NILs having the null genotype showed the greatest level of variation in eggs per gram of root, most likely owing to the reasons mentioned above. In contrast, variation

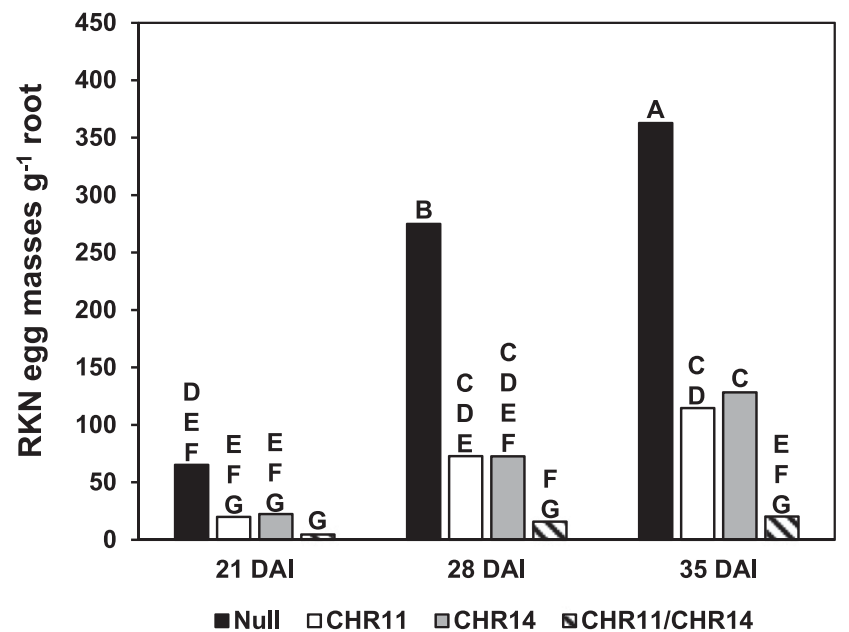

Fig. 2. Root-knot nematode (RKN) egg mass development on near isogenic lines (FiberMax-966 background) that are susceptible for $q M i-C 11$ and $q M i$ $\mathrm{Cl} 14$ (null), carry $q \mathrm{Mi}-\mathrm{C} 11$ alone (chromosome 11 [CHR11]), carry $q \mathrm{Mi}-\mathrm{Cl} 4$ alone (chromosome 14 [CHR14]), and carry both quantitative trait loci (CHR11/CHR14). Presented are the mean $(n=12)$ number of egg masses per gram of root (fresh weight) as determined at 21, 28, and 35 days after inoculation (DAI). Means having the same letter designation are not significantly different as determined using Fisher's protected least significant difference at $P \leq 0.05$. between NILs having the CHR11 or CHR14 genotypes was significantly less and NILs having both resistance loci were nearly identical.

At 7 DAI, CHR11, CHR14, and CHR11/CHR14 did not show any evidence of inhibited RKN J2 penetration of cotton roots or J2 migration within the root. This finding corroborates multiple studies that have demonstrated similar results (Creech et al. 1995; Da Silva et al. 2019; Shepherd and Huck 1989) and indicates that resistance does not hinge on reduced nematode attraction or preexisting physical barriers within the root tissues. However, clear differences in how CHR11 and CHR14 affected J2 development were apparent by 14 DAI. At 14 DAI, the J2 life stage persisted in comprising a significant percentage of the nematode population $(>60 \%)$ in CHR11 plants compared with CHR14 and null plants in which the $\mathrm{J} 2$ percentage dropped from nearly $100 \%$ at 7 DAI to only approximately $20 \%$ by 14 DAI. This decrease in J2 percentage in CHR14 and null plants at 14 DAI was complemented by a dramatic increase in the prevalence of RKN at the J3+J4 life stage. Da Silva et al. (2019) also observed a more rapid decline in $\mathrm{J} 2$ percentage in CHR14 and susceptible control lines. These observations indicate that CHR11 acts early in the infection process to hinder the development of sedentary $\mathrm{J} 2$ into later life stages or to prevent the establishment of migratory $\mathbf{J} 2$ at the outset.

We also investigated the effects of CHR 11 and CHR14 on RKN adult female fecundity measured as eggs/egg mass. When these resistance QTLs were combined into a single line, RKN fecundity was reduced by almost 4 -fold compared with null plants. This finding agrees with previous reports that utilized germplasm lines containing both resistance QTLs (Creech et al. 1995; Shepherd and Huck 1989). However, we discovered that CHR11 and CHR14 did not contribute equally to this phenotype. Although CHR11 appeared to reduce fecundity at 21 DAI compared with the null, this reduction was largely erased by $28 \mathrm{DAI}$, with eggs/egg mass being reduced only slightly compared with the null. In stark contrast, lines expressing only CHR14 showed eggs/egg mass means equivalent to those carrying both QTLs at both timepoints assayed. This finding indicates that CHR11-mediated RKN egg reduction is attributable for the most part to the overall decrease in nematodes that successfully establish a feeding site; although CHR14 lines may support a greater number of nematodes at later life stages,

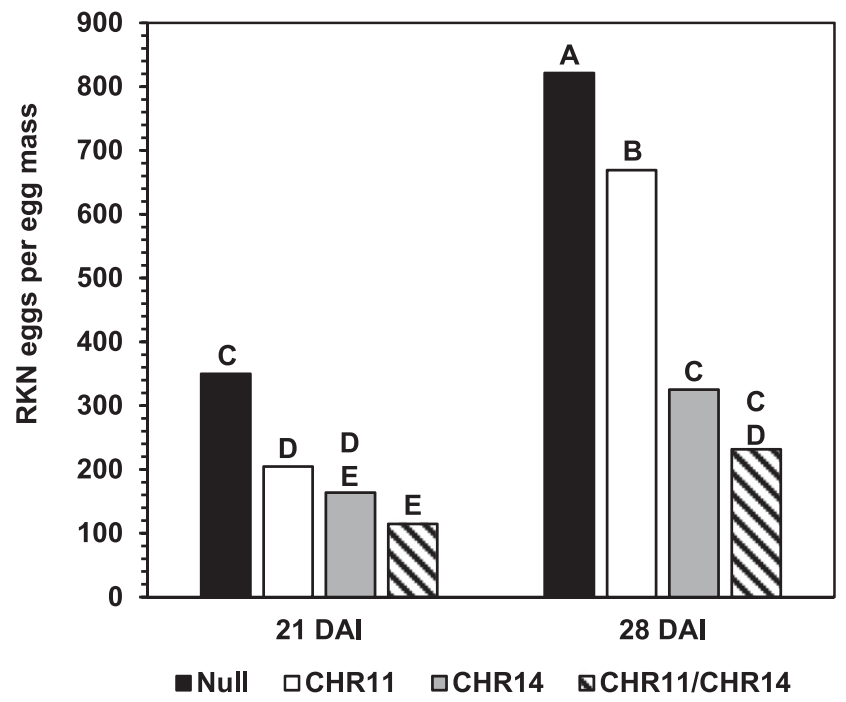

Fig. 3. Fecundity (eggs/egg mass) of adult female root-knot nematodes (RKNs) on near isogenic lines (FiberMax-966 background) that are susceptible for $q \mathrm{Mi}-\mathrm{Cl1}$ and $q \mathrm{Mi}-\mathrm{Cl} 4$ (null), carry $q \mathrm{Mi}-\mathrm{Cl1}$ alone (chromosome 11 [CHR11]), carry $q M i-C 14$ alone (chromosome 14 [CHR14]), and carry both quantitative trait loci (CHR11/CHR14). Presented are the mean $(n=15)$ number eggs/egg mass as determined at 21 and 28 days after inoculation (DAI). Means having the same letter designation are not significantly different as determined using Fisher's protected least significant difference at $P \leq 0.05$. 
they fail to progress into mature females and the females that develop are severely compromised in their ability to produce and lay eggs.

The causal gene underlying $q M i$-C14-mediated resistance was identified as Gh_D02G0276, which showed similarity to domesticated $h A T$-like transposases with the addition of novel $\mathrm{N}$ - and $\mathrm{C}$ terminal functional domains (Wubben et al. 2019). The D02G0276 allele that confers resistance showed a peculiar C-terminal truncation compared with the susceptible allele that resulted in a potentially antigenic domain owing to its identity with bacterial proteins (Wubben et al. 2019). Gene expression analyses showed that D02G0276 was slightly upregulated in resistant plants at 21 DAI, which coincides with the first indications of inhibited nematode development in the CHR14 NIL (Wubben et al. 2019). It is tempting to speculate that increased expression of the resistant D02G0276 allele late in the RKN life cycle triggers a cellular defense response that compromises further RKN development and shuts down egg production of mature females already present.

We discovered that both $q \mathrm{Mi}-\mathrm{Cl} 1 \mathrm{l}$ and $q \mathrm{Mi}-\mathrm{Cl} 4 \mathrm{reduced}$ the total number of RKN adult females. Given our findings of how these QTLs affected the postinfection development of RKN juveniles in different ways at different stages of development, it was not surprising to find that isolines expressing both QTLs showed a reduction in female numbers greater than either QTL alone. The differing mechanisms of resistance conferred by $q \mathrm{Mi}-\mathrm{Cl} 1$ and $q \mathrm{Mi}$ $C 14$ should provide cotton producers a relatively robust source of RKN resistance.

\section{LITERATURE CITED}

Creech, R. G., Jenkins, J. N., Tang, B., Lawrence, G. W., and McCarty, J. C. 1995. Cotton resistance to root-knot nematode: I. Penetration and reproduction. Crop Sci. 35:365-368.

Da Silva, M. B., Davis, R. F., Kumar, P., Nichols, R. L., and Chee, P. W. 2019. Resistance quantitative trait loci $q M i-C 11$ and $q M i-C 14$ in cotton have different effects on the development of Meloidogyne incognita, the southern root-knot nematode. Plant Dis. 103:853-858.

Gutiérrez, O. A., Jenkins, J. N., McCarty, J. C., Wubben, M. J., Hayes, R. W., and Callahan, F. E. 2010. SSR markers closely associated with genes for resistance to root-knot nematode on chromosome 11 and 14 of Upland cotton. Theor. Appl. Genet. 121:1323-1337.

He, Y., Kumar, P., Shen, X., Davis, R. F., Becelaere, G. V., May, O. L., Nichols, R. L., and Chee, P. W. 2014. Re-evaluation of the inheritance for root-knot nematode resistance in the Upland cotton germplasm line M-120 RNR revealed two epistatic QTLs conferring resistance. Theor. Appl. Genet. 127:1343-1351.

Hussey, R. S., and Barker, K. R. 1973. A comparison of methods of collecting inocula of Meloidogyne spp. including a new technique. Plant Dis. Rep. 57: 1025-1028.
Jenkins, J. N., Creech, R. G., Tang, B., Lawrence, G. W., and McCarty, J. C. 1995. Cotton resistance to root-knot nematode: II. Post-penetration development. Crop Sci. 35:369-373.

Jenkins, J. N., McCarty, J. C., Wubben, M. J., Hayes, R., and Gutiérrez, O. A. 2012. SSR markers for marker assisted selection of root-knot nematode (Meloidogyne incognita) resistant plants in cotton (Gossypium hirsutum L.). Euphytica 183:49-54.

Kirkpatrick, T. L., van Iersel, M. W., and Oosterhuis, D. M. 1995. Influence of Meloidogyne incognita on the water relations of cotton growth in microplots. J. Nematol. 27:465-471.

Lawrence, K., Hagan, A., Norton, R., Faske, T., Hutmacher, R., Mueller, J., Wright, D., Small, I., Kemerait, R., Overstreet, C., Price, P., Lawrence, G., Allen, T., Atwell, S., Jones, A., Thomas, S., Goldberg, N., Bowman, R., Goodson, J., Young, H., Woodward, J., and Mehl, H. 2017. Cotton disease loss estimate committee report, 2016. Pages 150-152 in Proceedings of the 2017 Beltwide Cotton Conference, vol. 1. National Cotton Council of America, Memphis, TN.

Lu, P., Davis, R. F., Kemerait, R. C., van Iersel, M. W., and Scherm, H. 2014. Physiological effects of Meloidogyne incognita infection on cotton genotypes with differing levels of resistance in the greenhouse. J. Nematol. 46:352-359.

McPherson, G. R., Jenkins, J. N., McCarty, J. C., and Watson, C. E. 1995. Combining ability analysis of root-knot nematode resistance in cotton. Crop Sci. 35:373-375.

McPherson, M. G., Jenkins, J. N., Watson, C. E., and McCarty, J. C. 2004. Inheritance of root-knot nematode resistance in M-315 RNR and M-78 RNR cotton. J. Cotton Sci. 8:154-161.

Mitchum, M. G., Hussey, R. S., Baum, T. J., Wang, X., Elling, A., Wubben, M., and Davis, E. L. 2013. Nematode effector proteins: An emerging paradigm of parasitism. New Phytol. 199:879-894.

Shen, X., Becelaere, G. V., Kumar, P., Davis, R. F., May, O. L., and Chee, P. 2006. QTL mapping for resistance to root-knot nematodes in the M-120 RNR Upland cotton line (Gossypium hirsutum L.) of the Auburn 623 RNR source. Theor. Appl. Genet. 113:1539-1549.

Shepherd, R. L. 1974. Transgressive segregation for root-knot nematode resistance in cotton. Crop Sci. 14:872-875.

Shepherd, R. L. 1982. Registration of three germplasm lines of cotton (Reg. Nos. GP 164-166). Crop Sci. 22:692.

Shepherd, R. L., and Huck, M. G. 1989. Progression of root-knot nematode symptoms and infection on resistant and susceptible cottons. J. Nematol. 21:235-241.

Shepherd, R. L., McCarty, J. C., Jenkins, J. N., and Parrott, W. L. 1996. Registration of nine cotton germplasm lines resistant to root-knot nematode. Crop Sci. 36:820.

U.S. Department of Agriculture. 2019. Cotton Varieties Planted 2019 Crop. Publication mp_cn833. U.S. Department of Agriculture Agricultural Marketing Service Cotton and Tobacco Program, Memphis, TN.

Williamson, V. M., and Hussey, R. S. 1996. Nematode pathogenesis and resistance in plants. Plant Cell 8:1735-1745.

Wubben, M. J., Thyssen, G. N., Callahan, F. E., Fang, D. D., Deng, D. D., McCarty, J. C., Li, P., Islam, M. S., and Jenkins, J. N. 2019. A novel variant of Gh_D02G0276 is required for root-knot nematode resistance on chromosome 14 (D02) in Upland cotton. Theor. Appl. Genet. 132:1425-1434.

Ynturi, P., Jenkins, J. N., McCarty, J. C., Gutiérrez, O. A., and Saha, S. 2006. Association of root-knot nematode resistance genes with simple sequence repeat markers on two chromosomes in cotton. Crop Sci. 46:2670-2674. 